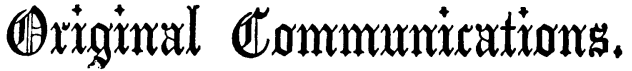

EVOLUTION OF THE FETUS IN UTERO.

By F. W. Mackenzie, M.D., M.R.C.P., Physician Accoucheur to the Paddington Maternity, etc.

[Read before the Medical Society of London, December 12th, 1859.]

THF particulars of the following case are submitted to the society for the purpose of showing that spontaneous evolution of the foetus, as described by Denman, does occasionally occur, and consequently that the doctrine of spontaneous expulsion, as maintained by Douglas and generally accepted by the pro. fession, must be received with some degree of qualification.

CASE. A lady, about five months advanced in her third pregnancy, was taken ill on the evening of the 1lth of November with symptoms of uterine uneasiness, but not such as to lead her to suspect that any danger of a miscarriage was imminent. On visiting her I found that she had experienced little or no pain, and had sustained little or no hæmorrhage; but on a vaginal examination $I$ found the membranes engaged in the os uteri, which was dilated to about the size of a shilling, and protruding into the vagina. Within half an hour the bag of membranes presented externally, and shortly afterwards broke. The liquor amnii, however, alone escaped, and nothing could be felt within the vagina, the fotus being as yet wholly in utero. After giving a few doses of ergot with cinnamon and bark, and waiting a short time, a spongy mass presented at the os uteri, which, after a few pains, was expelled, and found to be the placenta. No hæmorrhage followed upon its expulsion, and shortly afterwards, one of the superior extremities descended into the vagina, which, upon slight traction, broke away at the shoulder.joint. Convinced that manual assistance could be of little avail in the present state of the case, I left the patient for about two hours, and on then making an examination, found what proved to be the breech engaged in the os uteri. I followed it digitally downwards, and in due course the lower extremities descended; the case having assumed all the characters of an original breech-presentation. With a little manipulation the head was brought through the os uteri, and the entire birth completed without any difficulty. The patient made a good recovery, and throughout had scarcely any hæmorrhage.

Remarks. A brief reference to the views of Denman and Douglas respectively on the subject of "spontaneous evolution", will best indicate the relations of the present case to the main question at issue between them. By Denman it was maintained that "spontaneous evolution" consisted in a complete change in the position or evolution of the child in utero whereby some other part was substituted for that originally presenting; as for instance, the breech for the arm and shoulder. By Douglas, on the other hand, it was contended, that no such evolution or change in the position of the child could or did take place, and consequently that there could be no such substitution of one part of the child for another. $\mathrm{He}$ maintained that, in all the presumed cases of "evolution", in which the arm and shoulder originally presented and had descended into the pelvis, they remained unchanged in that position, whilst the body of the child was simultaneously doubled upon itself, and so forced by them through the pelvis. Now, there can be no doubt that this view is correct in regard to the greater number of instances which come under the designation of spontaneous evolution; but there are yet cases alleged to have occurred in which the evolution of the child, as described by Denman, has veritably taken place, and although perhaps rarely met with, are not on that account to be ignored. Dr. Murphy has met with such, and the present is one strictly to the point. Evolution of the fœtus, in the most rigid accepta. tion of the word, occurred; the breech was substituted for the arm, and from being originally an arm, it became eventually a breech-presentation. Undoubtedly, the early period of preg. nancy modifies very much the importance of the case in relation to the question at issue ; but it has, nevertheless, appeared to me worthy of record, as showing how, under certain circum. stances, spontaneous evolution may take place as described by Denman, and that his views, therefore, upon the subject, are not so wholly unsound as has been generally supposed. It is at least important, as showing that an argument which has been alleged against them is fallacious; viz., that it is incon- sistent with the nature of uterine contraction that such substitution of the presentation of one part of the child for another should take place.

\section{ON THE TREATMENT OF CONTRACTION OF THE KNEE FOLLOWING STRUMOUS AND RHEUMATIC DISEASES.}

By Holmes Сооте, Esq., F.R.C.S., Assistant-Surgeon to St. Bartholomew's Hospital, and to the Royal Orthopædic Hospital.

Some cases of disease of the knee-joint, illustrating the different methods of treatment necessary to extend a contracted limb, after the subsidence of all the acuter symptoms, have been lately under my care in St. Bartholomew's Hospital. Before mentioning the particulars, I would remark that, if cases the result of accident, or those proceeding from malignant disease, be omitted, the greater number of the remainder may be referred to one of those two conditions of constitution termed respectively " rheumatic" and "scrofulous".

In speaking, on a former occasion, of the rapid destruction of a joint by acute rheumatic inflammation, I was greatly surprised at finding the very existence of such a disease questioned by respectable authority. It may be useful, therefore, to repeat that such cases are unfortunately of too frequent occurrence. I can recall at the present moment the particulars of two, in which rapid disorganisation of the hip-joint occurred in sailors, both strong and active men; and, in the Museum of St. Bartholomew's Hospital, there is a preparation showing the usual morbid changes, of which case the following account is given. The patient was a sailor, forty years old. He awoke one night with intense pain in the hip, which lasted some hours, and then in a less degree continued till, in a fall, he bruised his hip. After this, the pain again became exceedingly severe; and this continued without remission till he died. Upon examination, it was found that the cartilage had been completely separated from the head of the femur, and from the acetabulum; and some shreds and ulcerated remnants of it were loose in the cavity of the joint. The ligamentum teres was destroyed. The exposed bones were superficially but smoothly ulcerated; the cavity of the acetabulum was enlarged by the ulceration of its walls. The capsule was thickened; the synovial surface swollen, soft, and thinly coated with lymph. (Ser. II, Prep. 14.)

The contrast between a joint disorganised by rheumatic in. flammation and that by scrofulous inflammation is well marked. In the former, the bones are of their normal weight and firmness, although the surface may be deeply ulcerated; in the latter, the bones are light, and give every indication of want of power. Hence it is that osseous anchylosis may be expected as a termination of rheumatic inflammation, but not of scrofulous disease; the only apparent exception to the above being those instances in which, after many years, a loose and spongy osseous tissue has been thrown out, the patient having outlived the strumous diathesis.

Preparations of osseous anchylosis of the knee are uncommon; they occur more frequently in the hip-joint. In the Museum of St. Bartholomew's Hospital there are but five preparations of the former. The first (Ser. II, Prep. 29) shows only bony union of the patella to the outer condyle of the femur. The second (Ser. II, Prep. 55) is in this respect precisely similar. The third (Subseries B, Prep. 22) exhibits more general osseous union. The fourth (Subseries B, Prep. 24) is a case of osseous union of the inner border of the femur to the corresponding surface of the tibia. The fifth (Subseries B, Prep. 26) is general osseous union, the bones being light and spongy. Similar cases are related by Bonnet (Malad. des Articul., t. ii, p. 201); by Löbl (Zeitschrift der $k$. $k$. Gesell. d. Aertze zu Wien, 1844); by Führer (Deutsche Klinik, 1850. Bd. 2, s. 429); by Astley Cooper, and by Sandefort and others. There are also some preparations in the College of Surgeons of England. But this list, so far from showing that similar preparations " hang about in dozens", proves directly the reverse. It is evidence that all such specimens are valu. able from their rarity.

It is scarcely possible to attach too much importance to this fact respecting osseous anchylosis of the knee, for it serves us as a guide in most important points of treatment. In incipient cases of strumous disease, we may safely predicate that 1015 